Gefässchirurgie 2018 $\cdot 23: 490-491$

https://doi.org/10.1007/s00772-018-0467-8

(C) Springer Medizin Verlag GmbH, ein Teil von Springer Nature 2018

CrossMark

\title{
F. Rohlffs
}

Klinik und Poliklinik für Gefäßmedizin, Universitäres Herzzentrum Hamburg, Klinik und Poliklinik für Gefäßmedizin, Universitätsklinikum Hamburg-Eppendorf, Hamburg, Deutschland

\section{Gefäßmalformationen}

Sehr geehrte Leserinnen, sehr geehrte Leser,

Gefäßmalformationen gelten als seltene Erkrankungen, und nur wenige Zentren weltweit verfügen über eine tiefgreifende Expertise mit langjähriger Erfahrung und hohen Eingriffszahlen auf diesem Gebiet. Das bedeutet für die betroffenen Patienten häufig eine Odyssee an Arztbesuchen, bis eine korrekte Diagnose gestellt oder eine Behandlung eingeleitet werden kann. Da eine Gefäßmalformation in verschiedenen Lebensabschnitten symptomatisch werden kann, ist man als betreuender Arzt mit Patienten aller Altersgruppen konfrontiert. Auch die klinische Präsentation dieser in der Regel angeborenen Erkrankung ist sehr variabel von asymptomatischen oberflächlichen Hautveränderungen hin zu lebensbedrohlichen Komplikationen wie Blutung und Herzinsuffizienz. Abhängig vom Typ der Gefäßmalformation (kapillär, venös, lymphatisch, arteriovenös, gemischt), der Angioarchitektur sowie ihrer Lokalisation umfasst die invasive Therapie endovaskuläre Strategien zur Embolisation der Malformation, Laserverfahren oder offen-chirurgische Techniken. Dies impliziert bereits die Vorteile eines interdisziplinären Teams zur Behandlung dieser komplexen Strukturen. Da ein offen-chirurgisches Vorgehen bei ausgedehnten Befunden mit einem großen Gewebetrauma assoziiert sein kann, sind endovaskuläre Techniken vorteilhaft. Allerdings wird die Wahl des Embolisats insbesondere in Bezug auf Nutzen und assoziierte Risiken lebhaft diskutiert. Zudem ist eine standardisierte Therapie am ehesten durch adäquate Klassifikationssysteme mit direkter Behandlungsimplikation zu erreichen.
In diesem Schwerpunktheft zum Thema Gefäßmalformationen ist es gelungen, international führende Experten (aus diesem Grund sind auch mehrere Artikel in englischer Sprache verfasst) zu gewinnen, um Vor- und Nachteile verschiedener Embolisate sowie die Wichtigkeit von Klassifikationssystemen mit direkten Behandlungsimplikationen zu eruieren. Durch die Einführung der Yakes-Klassifikation für arteriovenöse Malformationen (AVM) ist es zum Beispiel möglich, die AVM nach ihrer Struktur einzuteilen und die entsprechende Behandlungsstrategie direkt aus der Klassifikation abzuleiten. Dies ist ein großer Fortschritt in Hinblick auf eine standardisierte Therapie auch komplexer, vorher als nicht behandelbar eingeschätzter Läsionen, wie in dem Beitrag von Robert Vogelzang (Chicago, Illinois) eindrucksvoll dargestellt. Eine entsprechende Klassifikation existiert aber noch nicht im Bereich der venösen Malformationen, worüber der Beitrag von René Müller-Wille und Walter Wohlgemuth hervorragend informiert. $\mathrm{Zu}$ sätzlich wird das Problem inhomogener Nomenklaturen sowie der Einsatz von Eponymen anstatt einer klaren, klassifikationsbasierten Sprache im Umgang mit Malformationen benannt.

\section{》) Die klinische Präsentation von Gefäßmalformationen ist sehr variabel}

Der Beitrag von Wayne Yakes (Denver, Colorado) beleuchtet den in der aktuellen Literatur beschriebenen komplikationsarmen Einsatz von Äthanol als potentes Embolisat mit kurativem Potenzial im Vergleich mit anderen Substanzen, betont aber auch die erforderliche Er- 
fahrung des Anwenders. Es zeigt sich ein Defizit an Literatur bezüglich der langfristigen Okklusionsraten abhängig vom Typ des verwendeten Embolisates. Interessant ist hierzu die wegweisende Arbeit aus der Gruppe von Mary Meek (Little Rock, Arkansas). Die Autoren untersuchten anhand histologisch aufgearbeiteter Resektionspräparate von primär mit Onyx embolisierten Gefäßmalformationen die Rekanalisation von Onyx und die Bildung neuer Gefäßstrukturen im embolisierten Areal.

Mein Dank richtet sich an die hervorragenden Autoren, die alle sofort unterstützend ihre Mitwirkung an diesem Heft zugesagt haben. Sie alle zeichnen sich dadurch aus, Pioniere im Bereich der Gefäßmalformationen $\mathrm{zu}$ sein und leisten mit ihrem Engagement einen bedeutsamen Beitrag, um betroffenen Patienten zu helfen.

In diesem Sinne freuen wir uns, exzellente Beiträge präsentieren zu können, und wir hoffen, dass wir mit diesem Heft eine breite Leserschaft ansprechen können.

Herzliche Grüße,

Ihre

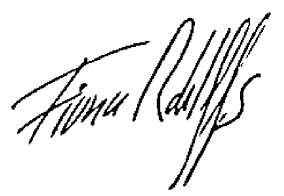

Dr. Fiona Rohlffs

\section{Korrespondenzadresse}

Dr. F. Rohlffs
Klinik und Poliklinik für
Gefäßmedizin, Universitäres
Herzzentrum Hamburg,
Klinik und Poliklinik
für Gefäßmedizin,
Universitätsklinikum
Hamburg-Eppendorf
Martinistraße 52, Gebäude
O50/O70, 20246 Hamburg,
Deutschland
f.rohlffs@uke.de

Interessenkonflikt. F. Rohlffs gibt an, dass kein Interessenkonflikt besteht.

Sadik Duru, Michael Gnant, Klaus Markstaller, Martin Bodingbauer Standards der OP-Patientenlagerung

Korrekte Lagerung und technische Ausstattung im modernen OP-Saal

Wien: Springer Verlag 2018, 1. Aufl., 194 S., 196 Abb., 17 Tab., (ISBN: 978-3-66257482-9 46), 46,25 EUR

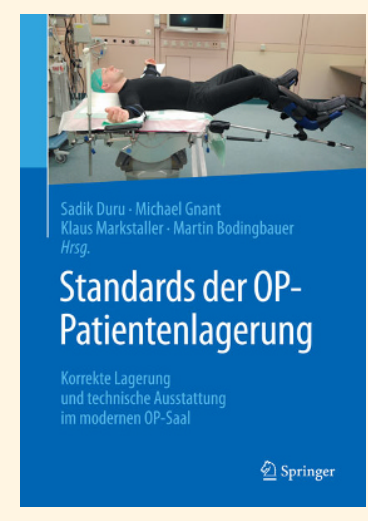

Komplexere und anspruchsvollere operative Eingriffe verlängern auch die Dauer mancher Operationen, laparoskopische und roboterassistierte Eingriffe sind mit besonderen Anforderungen an Übersichtlichkeit und Zugänglichkeit des Operationssitus verbunden. Damit muss auch der Patientenlagerung besondere Aufmerksamkeit zukommen. Folgeschäden nach erfolgreicher Operation aufgrund von Lagerungsfehlern beeinträchtigen das Endresultat empfindlich. Die OP-

Lagerung stellt also sowohl für die offene als auch für die minimal-invasive Chirurgie die Grundvoraussetzung für ein gutes Operationsergebnis dar. Sadik Duru, OP-Assistent im Allgemeinen Krankenhaus Wien, hat gemeinsam mit dem Leiter der Chirurgie, Univ. Klinik für Chirurgie, Prof. Dr. Michael Gnant, sowie mit dem Leiter der Anästhesie, Univ. Klinik für Anästhesie, Prof. Dr. Klaus Markstaller, und Dr. Martin Bodingbauer von der Univ. Klinik für Chirurgie die aktuellen Standards der OPPatientenlagerung im Springer-Verlag als Lehr - und Nachschlagewerk herausgegeben.

\section{Unterstützung für den Chirurgen - Schutz für den Patienten}

Die optimale Lagerung soll den Chirurgen in der Ausführung seiner Tätigkeit unterstützen, und den Patienten vor Schäden durch die Lagerung bewahren. „Lagerungsschäden können für den Patienten weitreichende Konsequenzen haben", unterstreichen die Herausgeber: „Sie reichen von Sensibilitätsstörungen bis hin zu Lähmungen, welche wiederum Invalidität und Arbeitsunfähigkeit zur Folge haben können. Das Resultat einer fehlerhaften Lagerung führt oft zu einem verlängerten Stationsaufenthalt, hinterlässt beim Patienten Ärger und Enttäuschung und hat häufig ein juristisches Nachspiel." Das lässt sich in vielen Fällen vermeiden. „Standards der OP-Patientenlagerung" gibt umfangreiche, detaillierte und klar strukturierte Empfehlungen, wie sich dies bei allgemeinchirurgischen, transplantationsspezifischen, gefäßchirurgischen, urologischen und gynäkologischen Eingriffen erreichen lässt. Dazu werden im speziellen Teil Indikationen, Vorgehen und Gefahren bei verschiedenen OPLagerungen, aufgegliedert nach Fachgebieten, mit vielen Bildern vermittelt.

\section{Die wichtigen Rahmenbedigungen: Von Hygiene bis zur Technik}

Der allgemeine Teil widmet sich den Rahmenbedingungen der OP-Situation: Vom Ziel einer einwandfreien Operationslagerung und den Erläuterungen zur perioperativen Medizin über die Hygiene im Operationsaal, rechtliche Aspekte der Patientenlagerung im $\mathrm{OP}$, die Grundlagerungsarten, modifizierte und abteilungsspezifische OP-Lagerungen bis zum Operationstisch, der Technik im OPSaal und schließlich Lagerungsschäden.

\section{Umfassende Zielgruppe}

Dieses Fachbuch richtet sich an Fachärzte und Ausbildungsassistenten der Chirurgie und Anästhesie, an operations-technische Assistenten und Pflegekräfte, die in Zusammenarbeit mit Chirurgen und Anästhesisten OP-Lagerungen vornehmen. 